\title{
The Effects of Clopidogrel and Omeprazole on Platelet Function in Normal Dogs
}

\author{
Brittany E. Thames, DVM, MPH, \\ Department of Clinical Sciences, College of Veterinary Medicine, Mississippi State University, \\ Mississippi State, MS 39762-6100 \\ Jennifer Lovvorn, BS, \\ Department of Clinical Sciences, College of Veterinary Medicine, Mississippi State University, \\ Mississippi State, MS 39762-6100
}

Mark G. Papich, DVM, MS, DACVCP, Department of Molecular Biomedical Sciences, College of Veterinary Medicine, North Carolina State University, Raleigh, NC 27607

Robert Wills, MS, DVM, PhD, DACVPM, Departments of Clinical Sciences and Pathobiology and Population Medicine, College of Veterinary Medicine, Mississippi State University, Mississippi State, MS 39762-6100

\section{Todd Archer, DVM, MS, DACVIM,}

Department of Clinical Sciences, College of Veterinary Medicine, Mississippi State University, Mississippi State, MS 39762-6100

Andrew Mackin, BVMS, MVS, DVSc, DACVIM, and Department of Clinical Sciences, College of Veterinary Medicine, Mississippi State University, Mississippi State, MS 39762-6100

John Thomason, DVM, MS, DACVIM

Department of Clinical Sciences, College of Veterinary Medicine, Mississippi State University, Mississippi State, MS 39762-6100

\section{Abstract}

Omeprazole is used concurrently with clopidogrel to reduce gastrointestinal adverse effects. In humans, the concurrent use of these two drugs can reduce the anti-platelet efficacy of clopidogrel. Our objective was to determine the effects of omeprazole and clopidogrel on platelet function in healthy dogs. A cross-over study utilized turbidimetric aggregometry (ADP and collagen) and the PFA-100® with the collagen/ADP cartridge to evaluate platelet function in eight healthy dogs during the administration of clopidogrel ( $1 \mathrm{mg} / \mathrm{kg} / 24 \mathrm{~h} \mathrm{PO})$, omeprazole $(1 \mathrm{mg} / \mathrm{kg} / 24 \mathrm{~h} \mathrm{PO})$, and a combination of clopidogrel and omeprazole. Drug metabolite concentrations were also measured. Compared to pretreatment, on Days 3 and 5, with ADP as the agonist, there was a significant decrease in maximum amplitude on aggregometry for both clopidogrel and clopidogrel/ omeprazole groups. The following revealed no significant differences between clopidogrel and

Corresponding Author: Dr. John Thomason, Department of Clinical Sciences, College of Veterinary Medicine, Mississippi State University, PO Box 6100, Mississippi State, 39762-6100; thomason@cvm.msstate.edu. 
clopidogrel/omeprazole groups when compared on Days 3 and 5: amplitude on aggegometry with ADP or collagen agonists, and PFA-100® closure times. When compared to the clopidogrel group, clopidogrel metabolite concentrations in the clopidogrel/omeprazole group were significantly higher on Days 3 and 5. The concurrent administration of omeprazole and clopidogrel in healthy dogs increased the plasma concentration of an inactive clopidogrel metabolite, but does not significantly alter the anti-platelet effects of clopidogrel.

\section{Keywords}

Canine; Aggregometry; Proton Pump Inhibitor; ADP Receptor Antagonist; PFA-100

\section{INTRODUCTION}

Thromboembolism is a devastating complication associated with several conditions in dogs, including immune-mediated hemolytic anemia, protein-losing nephropathy, atrial fibrillation, neoplasia, and parvoviral enteritis.(Brainard et al., 2010) Prophylactic medications are often administered to patients at risk of thromboembolism in order to inhibit either the clotting cascade or platelet function or both, in an effort to prevent thrombus formation. Unfortunately, many preventative anticoagulant medications can only be administered by injection or are cost prohibitive, typically leaving anti-platelet medication as the most affordable option for prophylactic therapy. Low-dose aspirin, the most widely used oral anti-platelet medication, does not consistently inhibit platelet function in all dogs. (Dudley et al., 2013; Haines et al., 2014) Other anti-platelet agents, particularly clopidogrel, have therefore more recently become popular for the prevention of thromboembolism in dogs.

Clopidogrel is a prodrug that requires hepatic metabolism to form an active metabolite that irreversibly blocks the platelet $\mathrm{P} 2 \mathrm{Y}_{12}$ receptor and inhibits platelet aggregation. (Boulenc et al., 2012; Ferreiro et al., 2010; Juurlink et al., 2009) In humans, the hepatic cytochrome P450 (CYP) enzymes, including CYP 2C19, CYP3A4, CYP1A2, and CYP2B, are responsible for clopidogrel metabolsm. However, the CYP 2C19 isoenzyme is believed to be the primary enzyme responsible for metabolism of clopidogrel.(Brainard et al., 2010; Mega et al., 2009) Unfortunately, it is unknown which enzyme is responsible for clopdogrel metabolism in dogs. In most dogs, when measured by aggregometry, platelet function is inhibited within 3 hours of clopidogrel administration, and platelet function remains inhibited for 24 hours after the first dose.(Brainard et al., 2010) Recently, a small prospective study evaluated the safety of clopidogrel in canine immune-mediated hemolytic anemia patients. When compared to low-dose aspirin, clopidogrel appeared to be an equally safe treatment option.(Mellett et al., 2011)

Clopidogrel therapy has, however, been previously associated with unwanted adverse effects in humans, including gastrointestinal hemorrhage.(Hallas et al., 2006; Tsai et al., 2012) Proton pump inhibitors, such as omeprazole, are therefore commonly administered with clopidogrel to reduce the risk and severity of gastrointestinal hemorrhage. Proton pump inhibitors, however, may inhibit the CYP 2C19 pathway that is needed to convert 
clopidogrel to its active metabolite in humans. (Collet et al., 2009; Kukanich et al., 2005; Mega et al., 2009) Concurrent administration of omeprazole and clopidogrel could therefore potentially reduce the metabolism of clopidogrel to its active form, and thereby limit the anti-platelet effects of clopidogrel. In humans, some studies have demonstrated that concurrent omeprazole reduces the anti-platelet effects of clopidogrel, and subsequently increases the risk of cardiovascular adverse events.(Bhatt et al., 2010; Cuisset et al., 2009; Juurlink et al., 2009; Siller-Matula et al., 2009) In fact, the FDA-approved label for human clopidogrel use states "Avoid concomitant administration of omeprazole with clopidogrel." Currently, it is not known if the concurrent administration of omeprazole and clopidogrel in dogs will cause similar interactions. Dogs do not possess the same CYP 2C19 enzyme as people,(Court, 2013) and it is not known if a similar enzyme is responsible for conversion of clopidogrel to its active form in dogs. The present study was designed to evaluate the effects of the concurrent administration of omeprazole and clopidogrel on platelet function and drug metabolite concentrations in normal dogs. Based on human studies, we hypothesized that when clopidogrel and omeprazole were administered concurrently in healthy dogs, the metabolism of clopidogrel to the active form would be reduced, subsequently decreasing the anti-platelet effects of clopidogrel.

\section{MATERIAL AND METHODS:}

\section{Study Design, Animals:}

Eight healthy Walker hound dogs, four intact females and four neutered males, were used in this study. The dogs were not exposed to any medications or vaccines for at least three weeks prior to initiation of the study. Normal health status was established by detection of no abnormalities on physical examination, complete blood count (including manual platelet count), serum biochemistry, urinalysis, and heartworm testing. The median age of the eight dogs was 1.5 years (range, 1-7 years), and their median body weight was $27.7 \mathrm{~kg}$ (range, $24.7-29.6 \mathrm{~kg}$ ). Study participants were weighed at the beginning of the study, and the weights obtained were used to calculate all subsequent doses. Animal use was approved by the Mississippi State University Institutional Animal Care and Use Committee, and was in compliance with the requirements at a facility accredited by the American Association for Accreditation of Laboratory Animal Care.

In a three way, randomized, cross-over study, the dogs were given either clopidogrel (clopidogrel bisulfate, Bristol-Myers Squibb, New York, NY) (1 mg/kg, PO, q24h), omeprazole (omeprazole, AstraZeneca, Wilmington, DE) (1 mg/kg, PO, q24h), or clopidogrel and omeprazole combination therapy $(1 \mathrm{mg} / \mathrm{kg}, \mathrm{PO}, \mathrm{q} 24 \mathrm{~h}$, and $1 \mathrm{mg} / \mathrm{kg}, \mathrm{PO}$, $\mathrm{q} 24 \mathrm{~h}$, respectively). All medications were administered orally for 5 days, followed by at least 14 days without any drug treatment. After this recovery period, the dogs switched groups, and the study was continued until all dogs had received each treatment.

Blood samples were collected for platelet function testing and measurement of the clopidogrel metabolite and omeprazole concentrations. There are no FDA-approved formulations for either drug available for dogs, and drug concentrations were therefore measured to ensure that oral absorption of both drugs occurred. For all drug combinations, blood samples were collected on Day 0 as a baseline before drug administration, and again 
on Days 3 and 5 of drug administration. For all medication groups, blood samples were collected two hours after drug administration. Blood samples were collected via jugular venipuncture with a 20 gauge needle directly into a vacutainer tube containing $3.2 \%$ sodium citrate anticoagulant or 143 units of sodium heparin.(Vacutainer tube, Becton Dickinson, Franklin Lakes, NJ) For assessment of platelet function, each sample was collected with a ratio of 1:9 citrate to blood to standardize the degree of anticoagulation.

\section{Platelet Function Analysis}

Turbidimetric Platelet Aggregometry-To create platelet-rich plasma (PRP), whole blood collected into $3.2 \%$ sodium citrate was centrifuged at $1,200 \times \mathrm{g}$ at room temperature for three minutes. The PRP supernatant was collected, and the remaining blood sample was centrifuged at $1,800 \times \mathrm{g}$ at room temperature for eight minutes to create platelet-poor plasma (PPP).

A two channel turbidimetric platelet aggregometer (Chronolog 700 Whole Blood/Optical Lumi-Aggregometer, Chronolog Corporation, Haverton, PA) was used to analyze platelet aggregation. For each dog, time point, and drug, three total samples were analyzed, and the results were averaged to yield a single value for each time point. Aggregation was assessed using ADP $(10 \mu \mathrm{M})$ or collagen $(10 \mu \mathrm{g} / \mathrm{mL})$ as agonists, with a temperature of $37^{\circ} \mathrm{C}$, and a stirring speed of 1,200 rpm. Samples were analyzed based on the manufacturer's standard guidelines (Chronolog 700 Manual, Chronolog Corporation, Haverton, PA). Briefly, $247 \mu \mathrm{l}$ of PRP was transferred into a glass cuvette containing a siliconized magnetic stir bar, and $250 \mu \mathrm{l}$ of PPP was placed into a cuvette without a stir bar. Samples were incubated, at $37^{\circ} \mathrm{C}$, for 1 minute, placed into the aggregometer, and stable baseline values corresponding to $0 \%$ and $100 \%$ aggregation were obtained using PRP and PPP, respectively. ADP or collagen were added to the PRP, and aggregation was monitored for 6 minutes and 8 minutes, respectively. The maximal percentage aggregation was calculated and recorded using computer software (AGGRO/LINK 8, Chronolog Corporation, Haverton, PA). Samples were maintained at room temperature (approximately $20-22^{\circ} \mathrm{C}$ ) without agitation prior to analysis. All samples were analyzed within 5 hours of collection. The number of platelets within the PRP was not adjusted to a standardized count by dilution with PPP prior to analysis, based on recommendations published by the International Society of Thrombosis and Haemostasis Platelet Physiology and Scientific and Standardization Committee. (Cattaneo et al., 2007; Linnemann et al., 2008; Mani et al., 2005)

Based on the established mechanism of action of the drug, measurement of ADP-induced platelet aggregation is considered to be the primary assessment of clopidogrel-induced inhibition of platelet function. Collagen-induced platelet aggregation is not necessarily a direct indicator of the effects of the drug on platelets, but does provide an overall assessment of platelet aggregation and function. Turbidimetric aggregometry, although traditionally considered the gold standard measure of platelet function, does not evaluate platelet function in whole blood or under shear forces that naturally occur in the patient. An additional measure of platelet function, a bench top platelet function analyzer using the same agonists, was therefore utilized to assess platelet function under more natural conditions. 


\section{Platelet Function Analyzer}

A whole blood platelet function analyzer (PFA-100®, Siemens Healthcare Diagnostics, Deerfield, IL), which has been validated for use in dogs, (Callan \& Giger, 2001; Nielsen et al., 2007; Morales et al., 2007) was used to analyze platelet function in whole blood. While passing blood under high shear forces, the PFA-100® measures the closure time, in seconds, that is required to form a platelet plug after activation with several agonists. The maximum closure time for the instrument is $>300$ seconds.

The instrument was used according to manufacturer's instructions. Briefly, blood samples were collected directly into blood collection tubes containing 3.2\% sodium citrate, and kept at room temperature without agitation until analysis. For analysis, $800 \mu \mathrm{l}$ of whole blood was transferred into a collagen/ADP cartridge (PFA Collagen/ADP Test Cartridge, Siemens Healthcare Diagnostics, Duluth, GA) and analyzed. Two collagen/ADP cartridges were analyzed at each time point for all dogs, and the closure times were averaged. All samples were analyzed within 2 hours of collection.

\section{Drug Concentration Analysis}

Clopidogrel is a prodrug that must be converted to an active metabolite (a thiol derivative) via formation of the 2-oxo-clopidogrel intermediate, followed by formation of the inactive carboxylic acid metabolite SR 26334. It is not feasible to measure the parent drug, clopidogrel, because it is rapidly converted to the active metabolite. Although the antiplatelet effects of clopidogrel are attributed to the active metabolite, measurement of the concentration of inactive metabolite SR 26334 has been used as a surrogate marker to verify oral absorption. In humans, SR 26334 represents $85 \%$ of the circulating clopidogrel metabolites.(Bahrami et al., 2008) For this study, the inactive clopidogrel metabolite was measured using a previously published and validated method for dogs.(Brainard et al., 2010) Omeprazole concentrations were measured using a previously validated assay for dogs. (Tolbert et al., 2011) For all drug assays, the acceptance criteria was the same as for the previously published assays. Quality control (QC) samples and fresh calibration samples were prepared each day. Calibration and QC samples were prepared in pooled blank (control) canine plasma collected from dogs prior to this study.

\section{Statistical Analysis}

Pre-study sample size calculations were performed using data from a previous study performed in humans.(Ferreiro et al., 2010) A sample size of 8 was calculated based on a goal of being able to detect a difference of greater than $20 \%$ in platelet function using an alpha of 0.05 and a power of 0.80 . Mixed models using PROC MIXED in SAS for Windows 9.4 (SAS for Windows version 9.2, SAS Institute, Cary, NC, 2008) were fit for each outcome that included day, treatment, and the day by treatment interactions as fixed effects. Dog identity was included as a random effect with variance components covariance structure. Repeated measures of dogs within run were accounted for in a repeated statement using an autoregressive one covariance structure. If the interaction term was significant, differences in least squares means between each of the levels of one variable were calculated for each level of the other variable in the interaction using an LSMESTIMATE statement. The simulate adjustment was used to compute p-values, adjusted for multiple comparisons, 
for outcomes with significant main effect or interaction terms. Plots of the residuals (the difference between the observed and predicted values) were assessed for each outcome to ensure the assumptions of the statistical method (e.g. normality and equal variance) had been met. An alpha level of 0.05 was used to determine statistical significance for all methods.

\section{RESULTS}

\section{Turbidimetric Aggregometry - ADP}

The median turbidimetric aggregometry maximum amplitude using ADP as an agonist for the omeprazole group on Day 0 was $62.1 \%$ (range, 25.7-80\%), while the median maximum amplitude on Day 3 and Day 5 was 50\% (range, 12-68.3\%) and 55.8\% (range, 22.7-65.6\%), respectively. There was no significant difference in maximum amplitude between Days 0, 3 and 5. The median maximum amplitude for the clopidogrel group on Day 0 was $63.3 \%$ (range, 30.3-73.3\%), while the median maximum amplitude on Day 3 and Day 5 was $11 \%$ (range, 2.3-16.3\%) and 7.5\% (range, 1.3-25\%), respectively. When compared to Day 0, there was a significant decrease in maximum amplitude on both Day $3(\mathrm{p}<0.0001)$ and Day 5 ( $p<0.0001$ ). There was no significant difference in maximum amplitude between Days 3 and 5. The median maximum amplitude for the clopidogrel/omeprazole group on Day 0 was $54.7 \%$ (range, 33-81.7\%), while the median maximum amplitude on Day 3 and Day 5 was $13.8 \%$ (range, 4-23\%) and 9.8\% (range, 0.3-29\%), respectively. When compared to Day 0, there was a significant decrease in maximum amplitude on both Day $3(\mathrm{p}<0.0001)$ and Day 5 ( $\mathrm{p}<0.0001)$. There was no significant difference in maximum amplitude between Days 3 and 5. There was no difference in the median maximum amplitude on Day 0 for any of the three medication groups. On Days 3 and 5, there was a significant decrease in maximum amplitude between the omeprazole group and the clopidogrel group, and the omeprazole group and the clopidogrel/omeprazole group. There was no difference in maximum amplitude between the clopidogrel group and the combined clopidogrel/omeprazole group. (Figure 1)

\section{Turbidimetric Aggregometry - Collagen}

The median turbidimetric aggregometry maximum amplitude using collagen as an agonist for the omeprazole group on Day 0 was 62\% (range, 53.7-81.3\%), while the median maximum amplitude on Day 3 and Day 5 was 53.7\% (range, 43-68.3\%) and 58.3\% (range, 46.3-70.7\%), respectively. The median maximum amplitude for the clopidogrel group on Day 0 was $62.8 \%$ (range, 55.3-75.7\%), while the median maximum amplitude on Day 3 and Day 5 was $46.3 \%$ (range, $28-59.3 \%$ ) and $42 \%$ (range, 29.7-67\%), respectively. The median maximum amplitude for the clopidogrel/omeprazole group on Day 0 was $57.8 \%$ (range, 42.7-80\%), while the median maximum amplitude on Day 3 and Day 5 was 36.3\% (range, 23.3-77.7\%) and 42.7\% (range, 2.3-75.3\%), respectively. Collectively, compared to Day 0, the maximum amplitude on Days 3 and 5 were significantly decreased, but was not dependent on a specific treatment group. There was no difference in maximum amplitude between Days 3 and 5. (Figure 2) 


\section{Platelet Function Analysis}

The median PFA-100® closure time (collagen/ADP cartridge) for the omeprazole group on Day 0 was 63 seconds (range, 52-109.5 seconds), while the median closure time on Day 3 and Day 5 was 67.8 seconds (range, 58.5-73.5 seconds) and 66.3 seconds (range, 58.5-188 seconds), respectively. The median closure time for the clopidogrel group on Day 0 was 69.3 seconds (range, 60.5-95.5 seconds), while the median closure time on Day 3 and Day 5 was 106.5 seconds (range, 53.5-196 seconds) and 133.5 seconds (range, 70-208), respectively. The median closure time for the clopidogrel/omeprazole group on Day 0 was 63.3 seconds (range, 53-80.5 seconds), while the median closure time on Day 3 and Day 5 was 78.5 seconds (range, 55-226 seconds) and 84.8 seconds (range, 66.5-300), respectively. Drug $(\mathrm{p}=0.0429)$ and day $(\mathrm{p}=0.0010)$ both had a significant effect on median closure time but the drug-day interaction was not significant $(\mathrm{p}=0.1965)$. There was a significant $(\mathrm{p}=0.0356)$ increase in the closure time between the omeprazole group and the clopidogrel group, however there were no difference between the omeprazole group and the clopidogrel/ omeprazole group and the clopidogrel group and the clopidogrel/omeprazole group. Collectively, compared to Day 0, the closure times on Days 3 and 5 were significantly increased, but was not dependent on a specific treatment group. There was no difference in closure times between Days 3 and 5. (Figure 3)

\section{Drug and Metabolite Concentrations}

The median plasma concentration of omeprazole in the omeprazole group was $0.83 \mu \mathrm{g} / \mathrm{ml}$ (range, $0.35-1.52 \mu \mathrm{g} / \mathrm{ml}$ ) on Day 3 and $0.67 \mu \mathrm{g} / \mathrm{ml}$ (range, 0.02-1.00 $\mu \mathrm{g} / \mathrm{ml}$ ) on Day 5, while the omeprazole concentration in the omeprazole and clopidogrel group was $0.82 \mu \mathrm{g} / \mathrm{ml}$ (range, $0.02-1.25 \mu \mathrm{g} / \mathrm{ml}$ ) and $0.85 \mu \mathrm{g} / \mathrm{ml}$ (range, below the limit of quantification - 1.52 $\mu \mathrm{g} / \mathrm{ml}$ ) on Days 3 and 5 respectively. On Day 0, the median plasma concentration of omeprazole in the omeprazole group was $0.03 \mu \mathrm{g} / \mathrm{ml}$ (range, $0.00-0.05 \mu \mathrm{g} / \mathrm{ml}$ ) and in the omeprazole/clopidogrel group was $0.03 \mu \mathrm{g} / \mathrm{ml}$ (range, $0.00-0.04 \mu \mathrm{g} / \mathrm{ml}$ ). In the omeprazole group, compared to Day 0, the concentration of omeprazole increased on Day 3 ( $\mathrm{p}<0.0001$ ) and Day 5 ( $\mathrm{p}<0.0001)$. There was no difference in omeprazole concentrations between Days 3 and 5. Additionally, there was no difference in the omeprazole concentrations between the omeprazole group and the clopidogrel/omeprazole group. (Figure 4A)

The median plasma concentration of the inactive carboxylic acid clopidogrel metabolite SR 26334 in the clopidogrel group was $0.24 \mu \mathrm{g} / \mathrm{ml}$ (range, $0.17-0.65 \mu \mathrm{g} / \mathrm{ml}$ ) on Day 3 and 0.23 $\mu \mathrm{g} / \mathrm{ml}$ (range, $0.13-0.28 \mu \mathrm{g} / \mathrm{ml}$ ) on Day 5 , while the clopidogrel metabolite concentration in the omeprazole and clopidogrel group was $0.50 \mu \mathrm{g} / \mathrm{ml}$ (range, $0.38-0.64 \mu \mathrm{g} / \mathrm{ml}$ ) and 0.47 $\mu \mathrm{g} / \mathrm{ml}$ (range, $0.41-0.85 \mu \mathrm{g} / \mathrm{ml}$ ) on Days 3 and 5 respectively. The plasma concentration of the clopidogrel metabolite in both groups on Day 0 was not detectable for all dogs. Compared to Day 0, in the clopidogrel group, the concentration of the clopidogrel metabolite increased on Days 3 ( $\mathrm{p}<0.0001)$ and 5 ( $\mathrm{p}<0.0001)$. In the clopidogrel/omeprazole group, compared to Day 0, the concentration of the cloidogel metabolite increased on Days 3 ( $\mathrm{p}<0.0001)$ and $5(\mathrm{p}<0.0001)$. Additionally, for both treatment groups, there was no difference in clopidogrel metabolite concentration between Days 3 and 5. However, when compared to the clopidogrel group, the clopidogrel metabolite concentration in the 
clopidogrel/omeprazole group was significantly greater on Day $3(\mathrm{p}<0.0001)$ and Day 5 $(\mathrm{p}<0.0001)$. (Figure 4B)

\section{DISCUSSION}

In 2009, the United States Food and Drug Administration released a statement warning that the concurrent administration of omeprazole with clopidogrel could decrease the antiplatelet effects of clopidogrel in humans.(Kwok \& Loke, 2012) Interestingly, although our study demonstrated that the concurrent administration of omeprazole with clopidogrel did not have a clinically relevant effect on the anti-platelet effects of clopidogrel in dogs, the addition of omeprazole did appear to have an effect on concentrations of the inactive clopidogrel metabolite. Specifically, administration of omeprazole was associated with significantly higher concentrations of the inactive clopidogrel carboxylic acid metabolite (SR 26334).

The initial studies performed in humans were based on results from platelet function assays, (Cuisset et al., 2009; Gilard et al., 2008), followed by studies evaluating clinical outcomes in hypercoagulable patients.(Bhatt et al., 2010; Ho et al., 2009; Juurlink et al., 2009; O’Donoghue et al., 2009) These studies have varying outcomes, creating considerable controversy regarding the impact of omeprazole on clopidogrel-associated platelet dysfunction. For example, from a systematic review of the effects of proton-pump inhibitors on the anti-platelet effects of clopidogrel, of nine studies assessing platelet function in patients receiving clopidogrel and omeprazole, five studies indicated a significant reduction in the anti-platelet effects of clopidogrel, while the remaining four studies demonstrated no significant effects.(Kwok \& Loke, 2012) The results of this study in dogs are consistent with these latter studies in humans, in that we did not demonstrate any significant difference in platelet function when dogs were treated with both clopidogrel and omeprazole compared to treatment with clopidogrel alone.

In our study, platelet function was evaluated via turbidimetric aggregometry (ADP or collagen as agonists) and the PFA-100® platelet function analyzer (collagen/ADP cartridge). In both human and veterinary medicine, platelet aggregometry is considered to be the gold standard method for evaluation of drug-induced platelet dysfunction.(Nielsen et al., 2007; Lordkipanidzé et al., 2009) Aggregometry assesses platelet function by using agonists to activate platelets, and measures platelet aggregation.(Lordkipanidzé et al., 2009) As expected, in our study, there was a significant decrease in platelet function following the administration of clopidogrel when ADP was the agonist, and a similar decrease in platelet function occurred when clopidogrel was administered concurrently with omeprazole. Additionally, for both the clopidogrel group and the clopidogrel/omeprazole group, there was a decrease in platelet function when collagen was used as the agonist. Our results are similar to studies in humans that identified a decrease in platelet aggregation both with clopidogrel and with a clopidogrel/omeprazole combination.(Ferreiro et al., 2010; O’Donoghue et al., 2009)

The second instrument used to assess platelet function in our study was the PFA-100®. This instrument uses whole blood under shear forces to assess platelet function, and is more user- 
friendly than aggregometry for the clinical setting. There is however some test-to-test variability in closure times generated by the PFA-100®. In order to compensate for this variability, two samples per dog per time point were analyzed and averaged. Our study demonstrated an increase in the PFA-100® closure times, suggesting a decrease in platelet function, when clopidogrel was administered, both with and without omeprazole. Similar to our aggregometry results, our PFA-100® results suggest that the concurrent administration of omeprazole with clopidogrel does not have a significant impact on the anti-platelet effects of clopidogrel. The PFA-100® collagen/ADP cartridge, however, may not be the ideal instrument and cartridge to assess clopidogrel-induced platelet dysfunction. In humans, the INNOVANCE® PFA P2Y cartridge has been reported to provide a better assessment of clopidogrel-associated platelet dysfunction than a standard PFA-100® Collagen/ADP cartridge.(Edwards et al., 2012; Lim et al., 2015; Tsantes et al., 2012) Unfortunately, this instrument and cartridge were not available at the time that our study was conducted.

The cause of the increase in concentrations of the inactive metabolite of clopidogrel associated with concurrent omeprazole administration detected in this study is undetermined. Clopidogrel is a prodrug and, after administration, must be converted to an active form that inhibits platelet function. In humans, there are two main pathways involved in clopidogrel metabolism. The first pathway creates the active metabolite, a thiol derivative, which leads to the anti-platelet effects of clopidogrel, while a second pathway forms the inactive carboxylic acid metabolite, SR 26334. In humans, the CYP enzymes, including CYP 2C19, CYP 3A4, CYP 1A2, and CYP 2B6, are responsible for the conversion of clopidogrel to the active metabolite.(Brainard et al., 2010) There is no evidence that dogs possess the CYP 2C19 enzyme (Court, 2013), and it is unknown if clopidogrel metabolism in dogs is similar to humans (Brainard et al., 2010; Chauret et al., 1997), but it possible that clopidogrel is metabolized through a similar CYP pathway. In people, omeprazole inhibits the CYP 2C19 enzyme, one of the enzymes that metabolizes clopidogrel, potentially interfering with the biotransformation that is responsible for the formation of the active metabolite of clopidogrel. Based on human studies, concurrent administration of omeprazole and clopidogrel, therefore, would be expected to produce lower blood concentrations of clopidogrel metabolites.

Clopidogrel and its active metabolite are usually undetectable in plasma using standard means, and the inactive carboxylic acid metabolite SR 26334 is therefore typically used as a surrogate to assess oral drug absorption.(Bahrami et al., 2008) In the dogs that received clopidogrel alone, our study revealed plasma concentrations of the inactive clopidogrel metabolite that were comparable to a previous study that evaluated the pharmacokinetic and pharmacodynamic effects of clopidogrel in dogs.(Brainard et al., 2010) When clopidogrel was administered concurrently with omeprazole, in contrast, the plasma concentration of the inactive clopidogrel metabolite nearly doubled. One possibility is that the concurrent administration of omeprazole inhibits the metabolism of clopidogrel to its active form, shunting the substrate instead to the inactive form. However, this would have been expected to have led to a smaller concentration of the active form of clopidogrel in the dogs receiving omeprazole, which in turn might have been expected to lead to impairment of the effects of clopidogrel on platelet function. This effect was not observed in this study. However, the lowest concentration of the active metabolite that consistently inhibits platelet function in 
dogs is unknown. According to Brainard et al., at a clopidogrel dose of $0.5 \mathrm{mg} / \mathrm{kg}$ daily, half of the dose used in the present study, platelet function in dogs was still effectively inhibited. (Brainard et al., 2010) Therefore, the $1 \mathrm{mg} / \mathrm{kg}$ daily clopidogrel dose used in our study may have produced sufficient concentration of active metabolite to adequately suppress platelet function, despite the shunting of more substrate to the inactive form. Future studies should explore the anti-platelet effects of the active clopidogrel metabolite if clopidogrel is administered at a lower dose concurrently with omeprazole.

There were several limitations to this study. First, this study was performed in healthy research dogs instead of hypercoagulable patients, and results may therefore not be directly applicable in clinical situations. Secondly, a study using a larger population may have been able to detect more subtle changes in platelet function. However, prior to initiating our study, we used the results of a human study(Ferreiro et al., 2010) to conduct a sample size calculation, and determined that eight dogs would be sufficient to determine if there was a clinically relevant difference (greater than 20\%) in platelet function when clopidogrel and omeprazole were combined. Although our study may not have had sufficient animals enrolled to detect subtle differences in platelet function, such differences, if they did exist but were not detected, would in our opinion not be enough to be clinically relevant. Thirdly, the tests of platelet function used in our study were similar to those used in some, but not all, comparable human studies. In human medicine, one of the factors contributing to the controversy regarding the effects of proton-pump inhibitors on clopidogrel-associated platelet dysfunction is a lack of standardization of the tests used for platelet function.(Kwok $\&$ Loke, 2012) Our study focused on platelet function and aggregation during the administration of these medication, however, other assays, such as flow cytometry and the assessment of the platelet phosphorylation state, as used in some human studies, could have been used to assess the effects of this drug combination on canine platelets. (Ferreiro et al., 2010; Siller-Matula et al., 2009) Finally, even though it is considered a surrogate marker, our study used the inactive metabolite of clopidogrel to represent drug metabolism, instead of the active metabolite. Unfortunately, in both human and veterinary medicine, it is extremely difficult to measure the active metabolite, requiring the use of this surrogate inactive metabolite instead.

In humans, it has been suggested that the concurrent use of clopidogrel and omeprazole can diminish the anti-platelet effects of clopidogrel, and that alternative gastrointestinal protectant drugs should therefore be considered. In contrast, our study found no evidence that the concurrent use of the two drugs significantly alters the anti-platelet effects of clopidogrel in healthy dogs. Although blood concentrations of an inactive clopidogrel metabolite increased when clopidogrel was administered concurrently with omeprazole, the clinical significance of this finding is undetermined.

\section{ACKNOWLEDGEMENTS}

The authors thank Matthew Raby and Cyndi Dunaway, Mississippi State University, for their technical assistance. Thanks to Delta R. Dise, Clinical Pharmacology Laboratory at North Carolina State University, for her assistance with drug analysis.

Funded by the Mississippi State University College of Veterinary Medicine Internal Competitive Research Grant, Dr. Hugh G. Ward Endowment, and National Institutes of Health (NIH 5T35OD010432-14). 


\section{REFERENCES}

Bahrami G, Mohammadi B, Sisakhtnezhad S. (2008) High-performance liquid chromatographic determination of inactive carboxylic acid metabolite of clopidogrel in human serum: Application to a bioequivalence study. Journal of Chromatography, 864, 168-172. [PubMed: 18295556]

Bhatt D, Cryer B, Contant C, Cohen M, Lanas A, Schnitzer T, Shook T, Lapuerta P, Goldsmith M, Laine L, Scirica B, Murphy S, Cannon C. (2010) Clopidogrel with or without omeprazole in coronary artery disease. New England Journal of Medicine, 363, 1909-1917. [PubMed: 20925534]

Boulenc X, Djebli N, Shi J, Perrin L, Brian W, Van Horn R, Hurbin F. et al. (2012) Effects of Omeprazole and Genetic Polymorphism of CYP2C19 on the Clopidogrel Active Metabolite. Drug Metabolism and Disposition, MD 2012; 40(1), 187-197.

Brainard BM, Kleine SA, Papich MG, Budsberg SCet al. (2010) Pharmacodynamic and pharmacokinetic evaluation of clopidogrel and the carboxylic acid metabolite SR 26334 in healthy dogs. American Journal of Veterinary Research, 71, 822-830. [PubMed: 20594086]

Callan MB, Giger U. (2001) Assessment of a point-of-care instrument for identification of primary hemostatic disorders in dogs. American Journal of Veterinary Research, 62, 652-658. [PubMed: 11341380]

Cattaneo M, Lecchi A, Zighetti ML, Lussana F. (2007) Platelet aggregation studies: autologous platelet-poor plasma inhibits platelet aggregation when added to platelet-rich plasma to normalize platelet count. Haematologica 92, 694-697. [PubMed: 17488697]

Chauret N, Gauthier A, Martin J, Nicoll-Griffith D. (1997) In vitro comparison of cytochrome p450mediated metabolic activities in human, dog, cat, and horse, Drug Metabolism And Disposition, 25, 1130-1136. [PubMed: 9321515]

Collet J, Hulot J, Pena A, Villard E, Esteve J, Silvain J, Payot L, Brugier D, Cayla G, Beygui F, Bensimon G, Funck-Brentano C, Montalescot G. (2009) Cytochrome P450 2C19 polymorphism in young patients treated with clopidogrel after myocardial infarction: a cohort study. Lancet, 373, 309-317. [PubMed: 19108880]

Court M (2013) Canine Cytochrome P-450 Pharmacogenetics. Veterinary Clinics of North America. Small Animal Medicine, 43, 1027-1038.

Cuisset T, Frere C, Quilici J, Poyet R, Gaborit B, Bali L, Brissy O, Morange P, Alessi M, Bonnet J. (2009) Comparison of omeprazole and pantoprazole influence on a high 150-mg clopidogrel maintenance dose: The PACA (Proton Pump Inhibitors And Clopidogrel Association) prospective randomized study. Journal of the American College of Cardiology, 54, 1149-1153. [PubMed: 19761935]

Dudley A, Thomason J, Fritz S, Grady J, Stokes J, Wills R, Pinchuk L, Mackin A, Lunsford K. (2013) Cyclooxygenase expression and platelet function in healthy dogs receiving low-dose aspirin. Journal of Veterinary Internal Medicine, 27, 141-149. [PubMed: 23278865]

Edwards A, Jakubowski J, Rechner A, Sugidachi A, Harrison P. (2012) Evaluation of the INNOVANCE PFA P2Y test cartridge: Sensitivity to P2Y12 blockade and influence of anticoagulant. Platelets, 23(2), 106-115. [PubMed: 21848368]

Ferreiro JL, Ueno M, Capodanno D, Desai B, Dharmashankar K, Darlington A, Charlton RK, Bass TA, Angiolillo DJ, (2010) Pharmacodynamic Effects of Concomitant Versus Staggered Clopidogrel and Omeprazole Intake Results of a Prospective Randomized Crossover Study. Circulation. Cardiovascular Interventions, 3, 436-441. [PubMed: 20858862]

Gilard M, Arnaud B, Cornily JC, Le Gal G, Lacut K, Le Calvez G, Mansourati J, Mottier D, Abgrall JF, Boschat J.et al. (2008) Influence of Omeprazole on the Antiplatelet Action of Clopidogrel Associated with Aspirin. Journal of the American College of Cardiology, 51(3), 256-260. [PubMed: 18206732]

Haines J, Thomason J, Bulla C, Seage E, Wills R, Lunsford K, Mackin A. (2014) In vitro and in vivo assessment of platelet function in healthy dogs during exposure to low-dose aspirin. Journal of Veterinary Internal Medicine, 28(3), 1051.

Hallas J, Dall M, Andries A, Andersen BS, Aalykke C, Hansen JM, Andersen M, Lassen AT. (2006) Use of single and combined antithrombotic therapy and risk of serious upper gastrointestinal 
bleeding: population based case-control study. British Medical Journal, 333, 726. [PubMed: 16984924]

Ho PM, Maddox TM, Wang Li, Fihn SD, Jesse RL, Peterson ED, Rumsfeld JS. (2009) Risk of Adverse Outcomes Associated With Concomitant Use of Clopidogrel and Proton Pump Inhibitors Following Acute Coronary Syndrome, Journal of the American Medical Association, 301, 937944. [PubMed: 19258584]

Juurlink DN, Gomes T, Ko DT, Szmitko PE, Austin PC, Tu JV, Henry DA, Kopp A, Mamdani MM. (2009) A Population-Based Study of the Drug Interaction Between Proton Pump Inhibitors and Clopidogrel. Canadian Medical Association Journal, 180(7), 713-718. [PubMed: 19176635]

Tsantes A, Ikonomidis I, Papadakis I, Kottaridi C, Tsante A, Kalamara E, Kardoulaki A, Kopterides P, Kapsimali V, Karakitsos P, Lekakis J, Travlou A. (2012) Evaluation of the role of the new INNOVANCE PFA P2Y test cartridge in detection of clopidogrel resistance. Platelets, 23(6), 481489. [PubMed: 22646492]

Kukanich B, Lascelles B, Aman A, Mealey K, Papich M. (2005) The effects of inhibiting cytochrome P450 3A, p-glycoprotein, and gastric acid secretion on the oral bioavailability of methadone in dogs. Journal of Veterinary Pharmacology and Therapeutics, 28, 461-466. [PubMed: 16207309]

Kwok CS, Loke YK. (2012) Effects of Proton Pump Inhibitors on Platelet Function in Patients Receiving Clopidogrel. Drug Safety, 2012; 35(2),: 127-139. [PubMed: 22204719]

Lim ST, Coughlan CA, Murphy S, Fernandez-Cadenas I, Montaner J, Thijs V, Marquardt L, McCabe D. (2015) Platelet function testing in transient ischaemic attack and ischaemic stroke: A comprehensive systematic review of the literature. Platelets, 26(5), 402-412. [PubMed: 26042726]

Linnemann B, Schwonberg J, Mani H, Prochnow S, Lindhoff-Last E. (2008) Standardization of light transmittance aggregometry for monitoring antiplatelet therapy: an adjustment for platelet count is not necessary. Journal of Thrombosis Haemostasis, 6, 677-683. [PubMed: 18182038]

Lordkipanidzé M, Pharand C, Schampaert E, Palisaitis DA, Diodati JG. (2009) Evaluation of the platelet count drop method for assessment of platelet function in comparison with "gold standard" light transmission aggregometry. Thrombosis Research, 124(4), 418-422. [PubMed: 19250657]

Mani H, Luxembourg B, Klaffling C, Erbe M, Lindhoff-Last E. (2005) Use of native or platelet count adjusted platelet rich plasma for platelet aggregation measurements. Journal of Clinical Pathology, 58, 747-750. [PubMed: 15976345]

Mega J, Close S, Wiviott S, Shen L, Hockett R, Brandt J, Walker J, Antman E, Macias W, Braunwald E, Sabatine M. (2009) Cytochrome P-450 Polymorphisms and Response to Clopidogrel. The New England Journal of Medicine, 360, 354-362. [PubMed: 19106084]

Mellett A, Nakamura R, Bianco D. (2011) A prospective study of clopidogrel therapy in dogs with primary immune-mediated hemolytic anemia. Journal of Veterinary Internal Medicine, 25, 71-75. [PubMed: 21155892]

Morales F, Couto C, Iazbik M. (2007) Effects of 2 concentrations of sodium citrate on coagulation test results, von Willebrand Factor concentration, and platelet function in dogs. Journal of Veterinary Internal Medicine, 21, 472-475. [PubMed: 17552453]

Nielsen L, Zois N, Pedersen H, Olsen L, Tarnow I. (2007) Platelet function in dogs: breed differences and effect of acetylsalicylic acid administration. Veterinary Clinical Pathology, 36, 267-273. [PubMed: 17806075]

O’Donoghue ML, Braunwald E, Antman EM, Murphy SA, Bates ER, Rozenman Y, Michelson AD, Hautvast RW, Ver Lee PN, Close SL, Shen L, Mega JL, Sabatine MS, Wiviott SD. (2009) Pharmacodynamic effect and clinical efficacy of clopidogrel and prasugrel with or without a proton-pump inhibitor: an analysis of two randomised trials. Lancet, 374, 989-997. [PubMed: 19726078]

Siller-Matula J, Spiel A, Lang I, Kreiner G, Christ G, Jilma B. (2009) Effects of pantoprazole and esomeprazole on platelet inhibition by clopidogrel. American Heart Journal, 157(1), 148e1-148e5. [PubMed: 19081411]

Tolbert K, Bissett S, King A, Davidson G, Papich M, Peters E, Degernes L. (2011) Efficacy of Oral Famotidine and 2 Omeprazole Formulations for the Control of Intragastric pH in Dogs. Journal of Veterinary Internal Medicine, 25, 47-54. [PubMed: 21143305] 
Tsai TJ, Lai KH, Hsu PI, Lin CK, Chan HH, Yu HC, Wang HM, Lin KH, Wang KM, Chang SN, Liu CP, Hsiao SH, Huang HR, Lin CH, Tsay FWet al. (2012) Upper gastrointestinal lesions in patients receiving clopidogrel anti-platelet therapy. Journal of the Formosan Medical Association 20,

$12 ; 111,: 705-710$. 


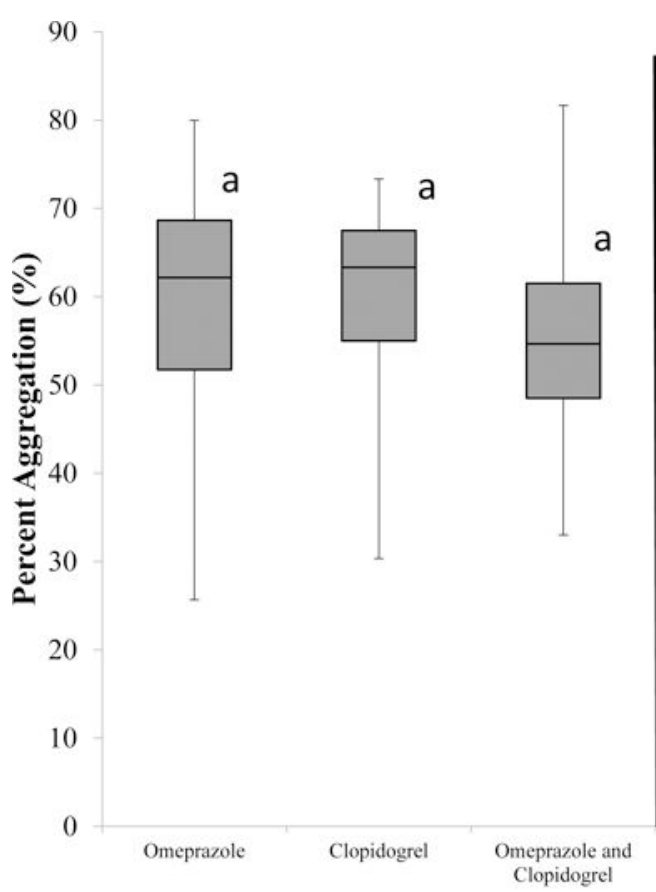

Day 0

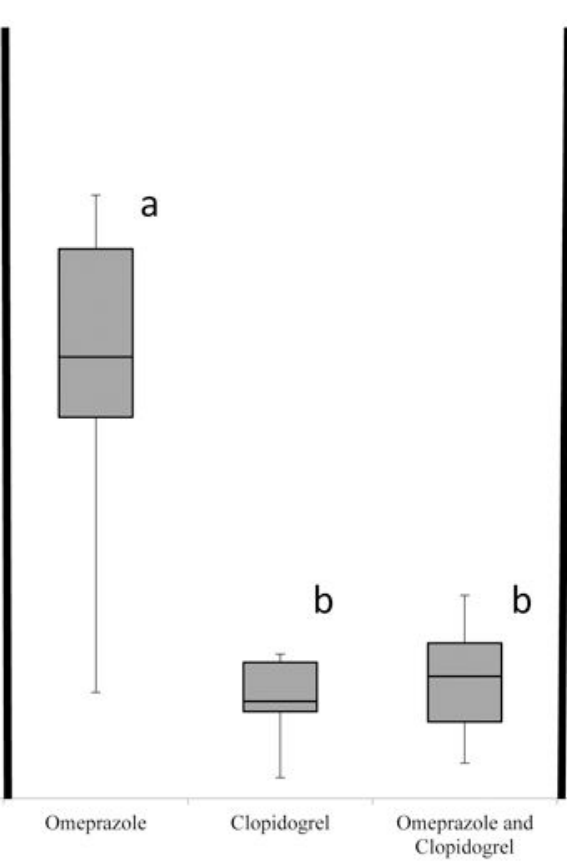

Day 3

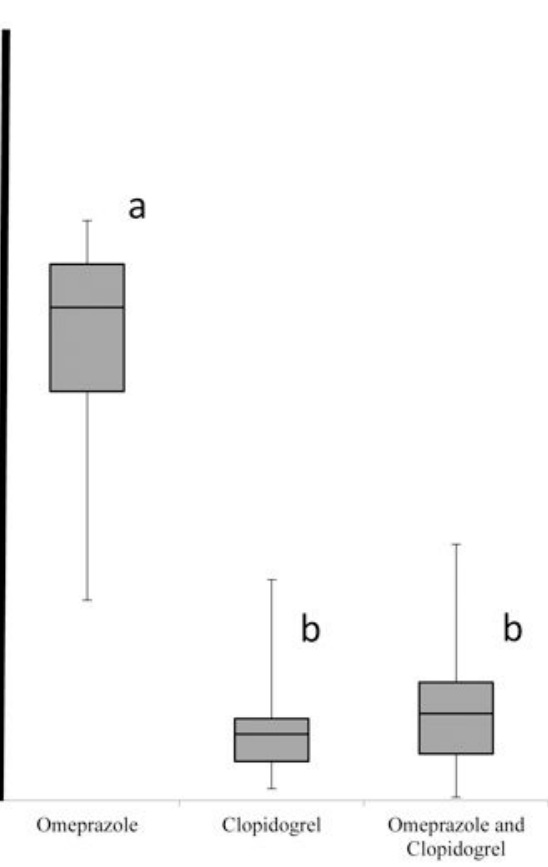

Day 5

Figure 1:

Maximum amplitude (percentage) of aggregation via turbidimetric aggregometry with ADP as the agonist in dogs treated with A. omeprazole (1 mg/kg, PO, q24h), B. clopidogrel (1 $\mathrm{mg} / \mathrm{kg}, \mathrm{PO}, \mathrm{q} 24 \mathrm{~h}$ ), and C. clopidogrel and omeprazole. The box and whiskers plot demonstrates the median (line), interquartile range (box), and total range (whiskers). Sampling times that do not share a letter are significantly $(\mathrm{p}<0.05)$ different. 


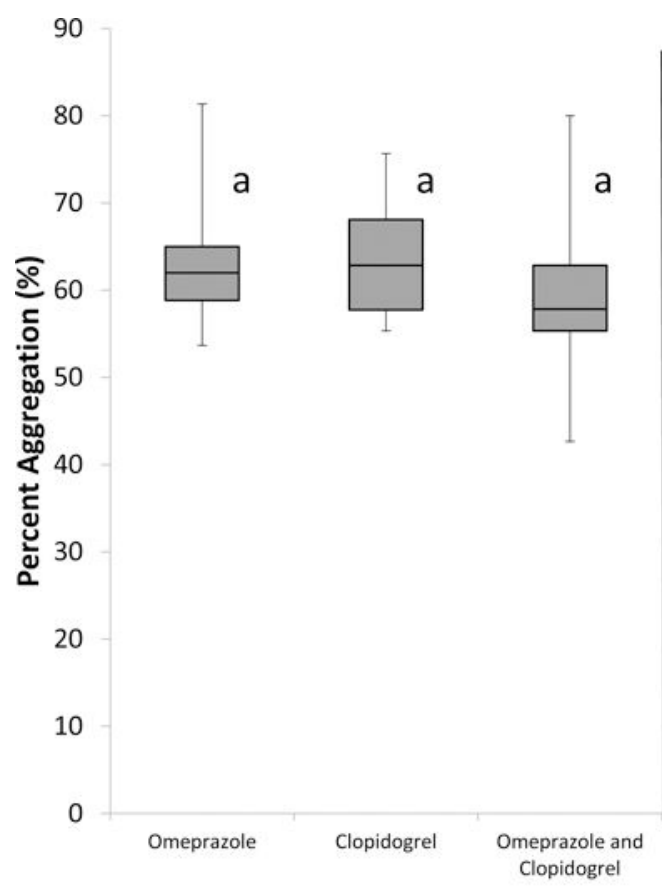

Day 0

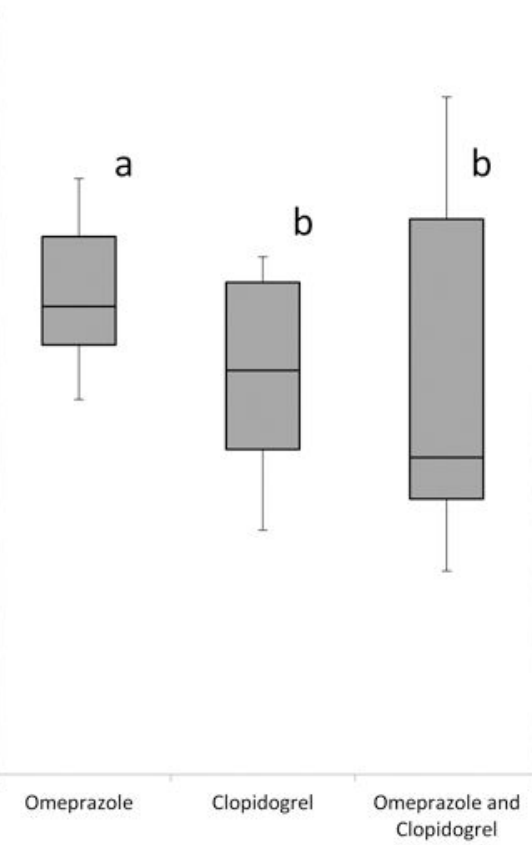

Day 3

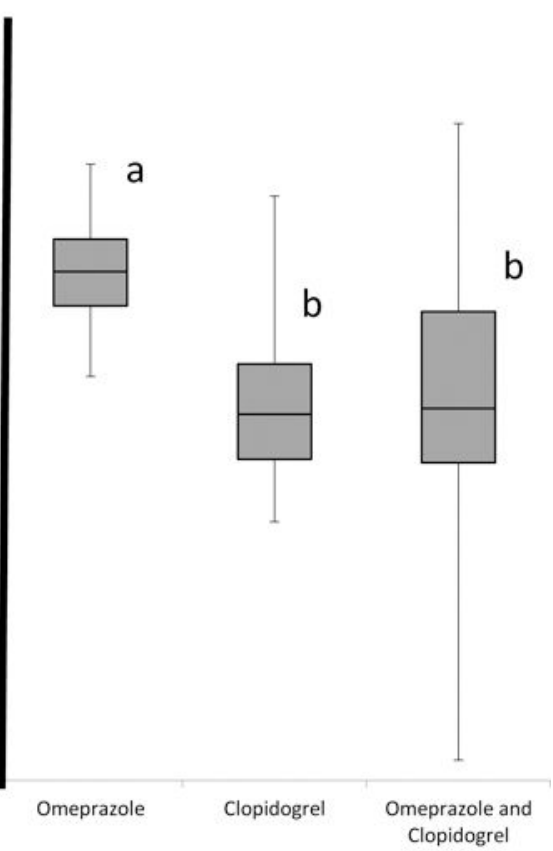

Day 5

Figure 2:

Maximum amplitude (percentage) of aggregation via turbidimetric aggregometry with collagen as the agonist in dogs treated with A. omeprazole, B. clopidogrel, and C. clopidogrel and omeprazole. Collectively, compared to Day 0, the maximum amplitude on Days 3 and 5 were significantly decreased. The box and whiskers plot demonstrates the median (line), interquartile range (box), and total range (whiskers). Sampling times that do not share a letter are significantly $(\mathrm{p}<0.05)$ different. 


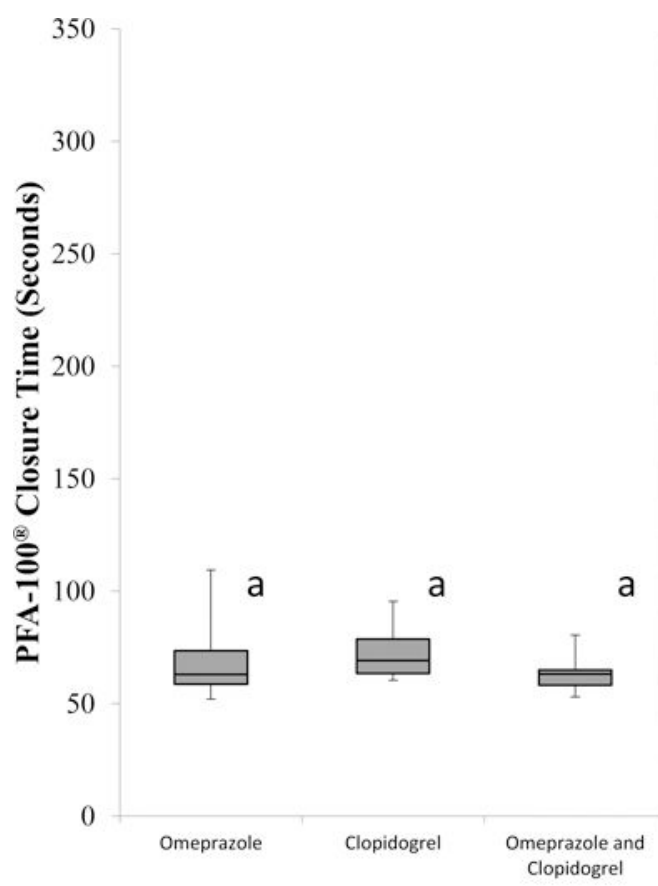

Day 0

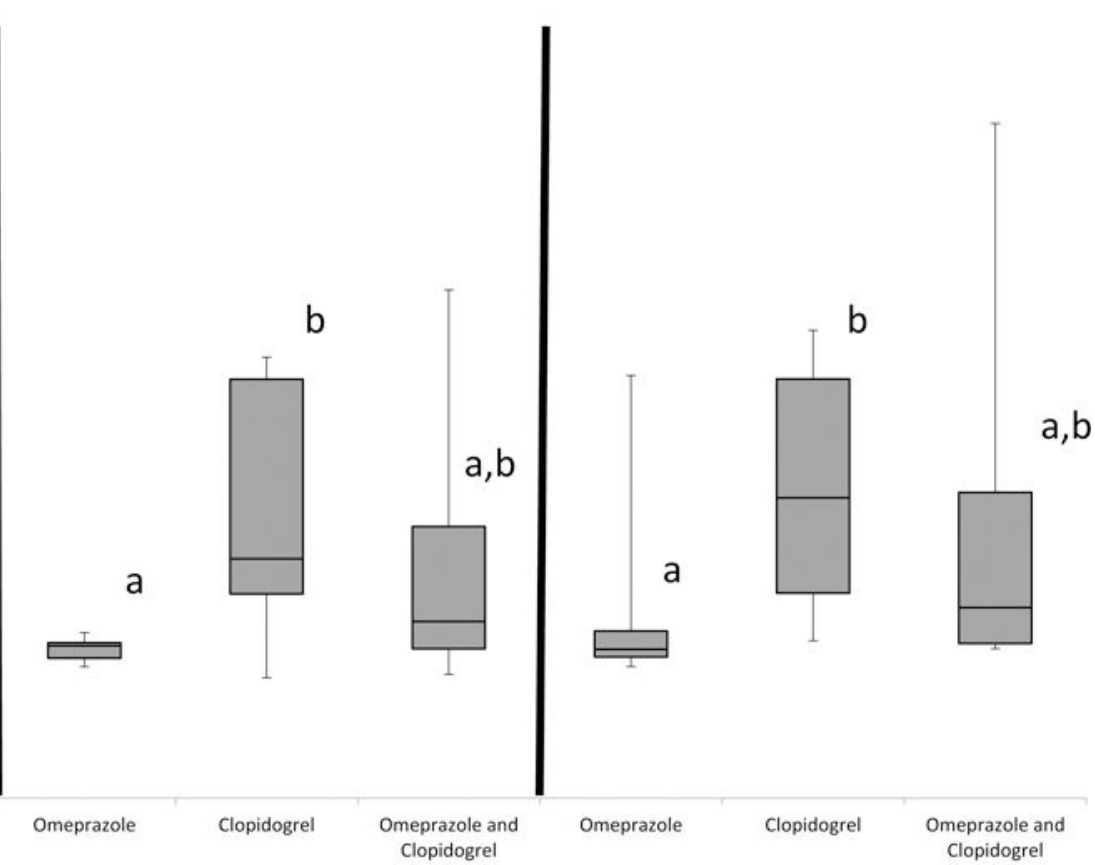

Day 3

Day 5

Figure 3:

PFA-100® closure times (collagen/ADP cartridge) in dogs treated with A. omeprazole, B. clopidogrel, and C. clopidogrel and omeprazole. Collectively, compared to Day 0 , the closure times on Days 3 and 5 were significantly increased. The box and whiskers plot demonstrates the median (line), interquartile range (box), and total range (whiskers). Sampling times that do not error share a letter are significantly $(\mathrm{p}<0.05)$ different. 

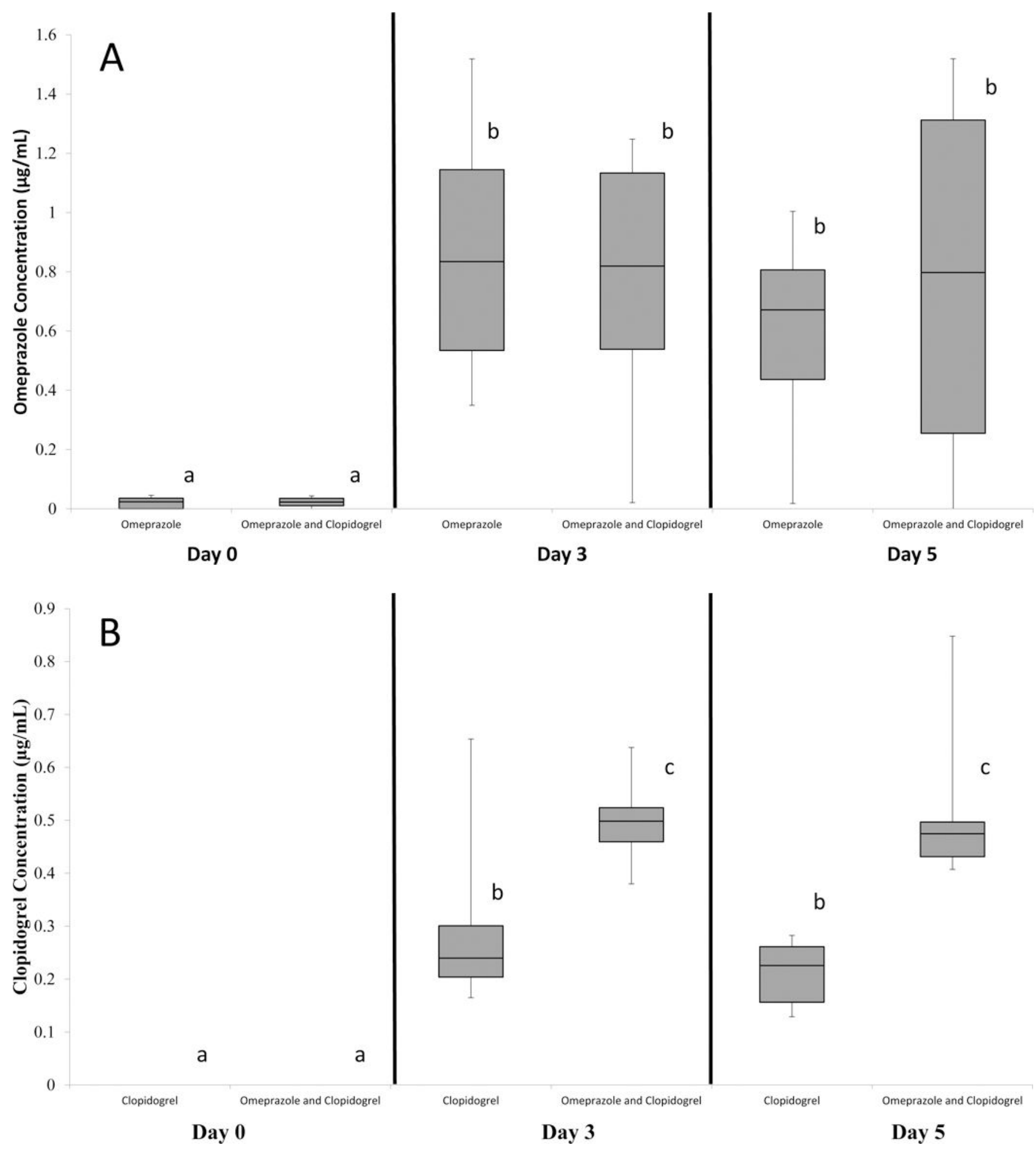

Figure 4:

Plasma concentration of A. omeprazole and B. the clopidogrel metabolite SR 26334 in dogs treated with 1. omeprazole, 2. clopidogrel, and 3. clopidogrel and omeprazole. The box and 
whiskers plot demonstrates the median (line), interquartile range (box), and total range (whiskers). Sampling times that do not share a letter are significantly $(\mathrm{p}<0.05)$ different. 